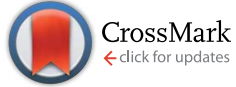

Cite this: RSC Adv., 2016, 6, 53903

Received 6th April 2016

DOI: $10.1039 / c 6 r a 08896 a$

www.rsc.org/advances
Accepted 25th May 2016

\section{Silanization as a versatile functionalization method for the synthesis of polymer/magnetite hybrid nanoparticles with controlled structure $\uparrow$}

\begin{abstract}
Alexander Schoth, ${ }^{a}$ Alasdair D. Keith, ${ }^{a}$ Katharina Landfester ${ }^{a}$ and Rafael Muñoz-Espi ${ }^{\star a b}$
We compare the use of different trimethoxysilane compounds for the surface functionalization of magnetite nanoparticles and their subsequent incorporation in hybrid particles formed by in situ polymerization. For the encapsulation of inorganic nanoparticles via miniemulsion polymerization, surface functionalization of the inorganic material is necessary to hydrophobize the otherwise hydrophilic inorganic material and to compatibilize it with the polymer. Hydrophobic magnetite nanoparticles are usually prepared by surface functionalization with oleic acid, which leads to effective hydrophobization, but offers only limited control over the structure of the hybrid particles. As an alternative, we report the encapsulation of magnetite particles functionalized with 3-methacryloxypropyl trimethoxysilane (MPS) or octadecyl trimethoxysilane (ODTMS). The influence of the surface functionalization on the compatibility of the inorganic particles with different polymers is investigated by determining the solid content of the dispersions and the magnetite content of the hybrids. The morphology of the hybrid materials is studied by transmission and scanning electron microscopy. MPSfunctionalized magnetite particles are distributed homogeneously within the polymers, while ODTMS and oleic acid lead to the formation of Janus particles. These observations are similar regardless of the polymer, which demonstrates that the functionalization is the decisive factor in tuning the structure of hybrid nanoparticles.
\end{abstract}

\section{Introduction}

Inorganic nanomaterials are commonly used in a broad range of applications. The use of silica as a filler material to increase mechanical strength, ${ }^{\mathbf{1}}$ titania because of its UV absorption ability, ${ }^{2}$ and magnetite as a contrast agent in magnetic resonance imaging (MRI) ${ }^{3,4}$ are some typical examples. In all of these cases, the use of the pure inorganic material might be problematic because it is not trivial to achieve the desired colloidal stability or biocompatibility. ${ }^{3,5}$ Encapsulation of the inorganic particles in a polymeric material can help to overcome these problems. Methods like dispersion polymerization $^{6-8}$ or seeded emulsion polymerization ${ }^{9-11}$ are often used to encapsulate inorganic nanoparticles, but a major drawback of these techniques is the limitation of the possible structures, ${ }^{\mathbf{1 2}}$ since most of the strategies deliver either a polymer particle surrounded by inorganic particles ${ }^{\mathbf{1 3}}$ or an inorganic particle with a polymer shell. ${ }^{14}$

${ }^{a}$ Max Planck Institute for Polymer Research, Ackermannweg 10, 55128 Mainz, Germany

${ }^{b}$ Institute of Materials Science (ICMUV), Universitat de València, c/Catedràtic José Beltrán 2, 46980 Paterna, Spain.E-mail: rafael.munoz@uv.es

$\uparrow$ Electronic supplementary information (ESI) available: Additional TGA data. See DOI: $10.1039 / \mathrm{c} 6 \mathrm{ra0} 8896 \mathrm{a}$
Miniemulsion polymerization has been shown to be a convenient method to synthesize polymer/inorganic hybrid nanoparticles with a controlled structure. ${ }^{15-17}$ Miniemulsion droplets are kinetically stabilized by the addition of an osmotic reagent, and a relatively low amount of surfactant is needed. ${ }^{\mathbf{1 8}}$ Nanomaterials produced in miniemulsion are, therefore, suitable for biomedical applications, such as encapsulated magnetite used as a contrast agent. ${ }^{19}$ A large variety of inorganic materials can be encapsulated by miniemulsion, including silica, ${ }^{20,21}$ magnetite, ${ }^{4,22}$ titania, ${ }^{23}$ and gold. ${ }^{24}$ Among other monomers, methyl methacrylate (MMA), ${ }^{20}$ styrene, ${ }^{25}$ and lactic acid $^{26}$ have been used. To determine the structure of the hybrid particles, the overall interfacial energy of the system has to be minimized. ${ }^{12,27}$ The interfacial energy can be tuned by changing the type of initiator ${ }^{4,28}$ or the surfactant concentration, ${ }^{29}$ so that a certain structure is favored. Other important parameters are the polarities of the polymer and the inorganic particles, as they determine the interfacial energies between these materials and the aqueous phase..$^{12,28}$ The surface properties of the inorganic material can be easily tuned by surface functionalization. For magnetite nanoparticles, oleic acid is one of the most common functionalization agents. ${ }^{30}$ Carboxylic acids act as chelating ligands for iron atoms and form stable bonds. Even if widely used, the encapsulation of oleic-acid-functionalized magnetite particles in miniemulsion is still challenging in terms of 
morphological control, as different publications show. ${ }^{22,31-33}$ In most cases, a combination of miniemulsion and emulsion polymerization is applied. In the first step, a magnetite cluster is prepared in miniemulsion, followed by emulsion polymerization around the cluster. ${ }^{34-36}$

As oleic-acid-functionalized particles are very hydrophobic, they often form nanoparticles with Janus-like structures. ${ }^{31,33}$ To obtain a homogeneous distribution of the magnetite inside the polymer, the system has to be tuned. Mori et al., for example, achieved this by changing from oil-soluble to water-soluble initiators. ${ }^{4}$ Another possibility is to use trialkoxysilanes instead of oleic acid as a functionalization agent. The silane chemistry is a versatile technique with a huge variety of accessible functional groups. ${ }^{37}$ The encapsulation of surfacefunctionalized silica particles in different polymers is an established technique to control the structure of the hybrid material. ${ }^{12,16,21}$ However, for magnetite, silanization has been mostly used to tune the magnetic properties ${ }^{38,39}$ or the interaction with biomaterials, but not to control the morphology. ${ }^{\mathbf{4 0}-42}$

We report here the synthesis of polymer/magnetite hybrid nanoparticles with a controlled structure by using miniemulsion polymerization. The magnetite particles have been functionalized with 3-methacryloxypropyl trimethoxysilane (MPS), octadecyl trimethoxysilane (ODTMS), and oleic acid (OA). Encapsulation experiments have been done in methyl methacrylate (MMA), styrene, and a mixture of styrene and 4vinylpyridine (4VP). The compositions of the hybrid particles have been determined by thermogravimetric analysis (TGA). To observe the inner structure of the hybrids, transmission and scanning electron microscopy (TEM and SEM) have been applied.

\section{Experimental section}

\section{Materials}

Methyl methacrylate (MMA, >99\%, Sigma-Aldrich) and styrene (>99\%, Sigma-Aldrich) were passed through an alumina chromatography column before use to remove the inhibitor. 4Vinylpyridine (4VP, 95\%, Sigma-Aldrich) was distilled under reduced pressure. Hexadecane ( $>99 \%$, Sigma-Aldrich), the oilsoluble initiator 2,2'-azobis(2-methylbutyronitrile) (V59, Wako Chemicals), sodium dodecyl sulfate (SDS, Alfa Aesar), absolute ethanol (Fluka), ammonium hydroxide (28\% solution in water, VWR), oleic acid (Sigma-Aldrich), methacrylic acid (99\%, SigmaAldrich), 3-methacryloxypropyl trimethoxysilane (MPS, 98\%, Sigma-Aldrich), octadecyl trimethoxysilane (ODTMS, 90\%, Sigma-Aldrich), ferric chloride hexahydrate $\left(\mathrm{FeCl}_{3} \cdot 6 \mathrm{H}_{2} \mathrm{O}, 99 \%\right.$, Acros), and ferrous chloride tetrahydrate $\left(\mathrm{FeCl}_{2} \cdot 4 \mathrm{H}_{2} \mathrm{O}, 99 \%\right.$, Merck) were used without further purification.

\section{Synthesis of acid-functionalized magnetite nanoparticles}

Acid-functionalized magnetite nanoparticles were synthesized according to a slightly varied procedure from those previously reported. ${ }^{22,36}$ Ferrous chloride $(3.0 \mathrm{~g}, 15 \mathrm{mmol})$ and ferric chloride $(6.1 \mathrm{~g}, 22.5 \mathrm{mmol})$ were dissolved in $20 \mathrm{~mL}$ of water. A concentrated ammonia solution $(10 \mathrm{~mL})$ was diluted with water
(5 $\mathrm{mL}$ ) and added to the iron salt solution dropwise within 5 min under vigorous mechanical stirring. Either oleic acid or methacrylic acid $(3.5 \mathrm{mmol})$ was added and, afterwards, the reaction mixture was first heated to $70{ }^{\circ} \mathrm{C}$ for $1 \mathrm{~h}$ and then to $130{ }^{\circ} \mathrm{C}$ for $2 \mathrm{~h}$. The obtained magnetite particles were washed with water several times and dried under vacuum.

\section{Synthesis of silanized magnetite nanoparticles}

Ferrous chloride (3.0 g, $15 \mathrm{mmol})$ and ferric chloride (6.1 g, $22.5 \mathrm{mmol}$ ) were dissolved in $20 \mathrm{~mL}$ of water. A concentrated ammonia solution $(10 \mathrm{~mL})$ was diluted with water $(5 \mathrm{~mL})$ and added to the iron salt solution dropwise within 5 min under vigorous mechanical stirring. Afterwards, the reaction mixture was first heated to $70{ }^{\circ} \mathrm{C}$ for $1 \mathrm{~h}$ and then to $130{ }^{\circ} \mathrm{C}$ for $2 \mathrm{~h}$. The supernatant solution was removed after collecting the particles with a magnet, which were redispersed in a mixture of water $(100 \mathrm{~mL})$, ethanol $(50 \mathrm{~mL})$ and SDS (30 mg). The $\mathrm{pH}$ of the mixture was set to 9.5 using concentrated ammonia solution. The silane compound (MPS or ODTMS, $20 \mathrm{mmol}$ ) was added dropwise within $30 \mathrm{~min}$ and the mixture was stirred at ambient temperature for another hour. After heating to reflux at $105{ }^{\circ} \mathrm{C}$ for $1.5 \mathrm{~h}$, the particles were washed several times with a water/ethanol mixture $(1: 1)$ and then dried under vacuum.

\section{Synthesis of hybrid nanoparticles}

The organic phases were prepared by dissolving hexadecane $(100 \mu \mathrm{L})$ and V59 $(50 \mathrm{mg})$ in the different monomers. The samples contained MMA $(1.8 \mathrm{~mL})$, styrene $(1.8 \mathrm{~mL})$ or a mixture of styrene $(1.4 \mathrm{~mL})$ and $4 \mathrm{VP}(0.4 \mathrm{~mL})$. Magnetite particles (200 $\mathrm{mg}$ ) were added to the samples and dispersed in the ultrasound bath for $30 \mathrm{~min}$. After addition of the aqueous phase consisting of water $(18 \mathrm{~mL})$ and SDS (36 mg), the emulsions were shaken for $20 \mathrm{~min}$. Ultrasonication for $120 \mathrm{~s}$ at $70 \%$ intensity with a pulse sequence of $30 \mathrm{~s}$ pulse and $10 \mathrm{~s}$ pause (Branson W 450 digital sonifier; $1 / 2^{\prime \prime}$ tip, ice cooling) was used to prepare the miniemulsions. Polymerization took place at $72{ }^{\circ} \mathrm{C}$ for $17 \mathrm{~h}$ in a thermoshaker. The polymerized samples were then filtered to remove the possibly formed coagulum.

Separation of the particles containing magnetite from the empty polymer particles was performed by putting the dispersions on a neodymium magnet for $30 \mathrm{~min}$. The supernatant dispersion was removed and the particles were redispersed in a $0.1 \mathrm{wt} \%$ SDS solution $(10 \mathrm{~mL})$. The dispersion was again purified magnetically and then refilled with water. The solid content of the samples was determined by lyophilization.

\section{Characterization techniques}

Particle sizes and size distributions were determined by dynamic light scattering (DLS) using a Nicomp particle sizer 380 (PSS) at a fixed angle of $90^{\circ}$.

The magnetite content of the lyophilized hybrid particles was determined using thermogravimetric analysis (TGA). The measurements were conducted using a Mettler-Toledo TGA/ SDTA-851 thermobalance (40 to $700{ }^{\circ} \mathrm{C}$, heat rate of $10 \mathrm{~K}$ $\min ^{-1}$, nitrogen atmosphere). 
For scanning electron microscopy, the diluted samples were drop-casted on a silicon wafer. The micrographs were recorded using a Leo Gemini 1530 field emission microscope at an extractor voltage of $0.5 \mathrm{kV}$.

Transmission electron microscopy (TEM) was measured on a JEOL JEM-1400 electron microscope at an acceleration voltage of $120 \mathrm{kV}$. The samples were prepared by drop-casting of the diluted dispersions on a carbon-coated copper grid. The samples containing PMMA were coated with a thin carbon layer before the measurement.

\section{Results and discussion}

Inorganic nanoparticles are usually hydrophilic, while many common polymers are hydrophobic. To enable encapsulation of inorganic particles in such hydrophobic polymers, the compatibility of the two materials has to be improved, which is mostly achieved by surface functionalization of the inorganic material. For magnetite nanoparticles, capping with oleic acid

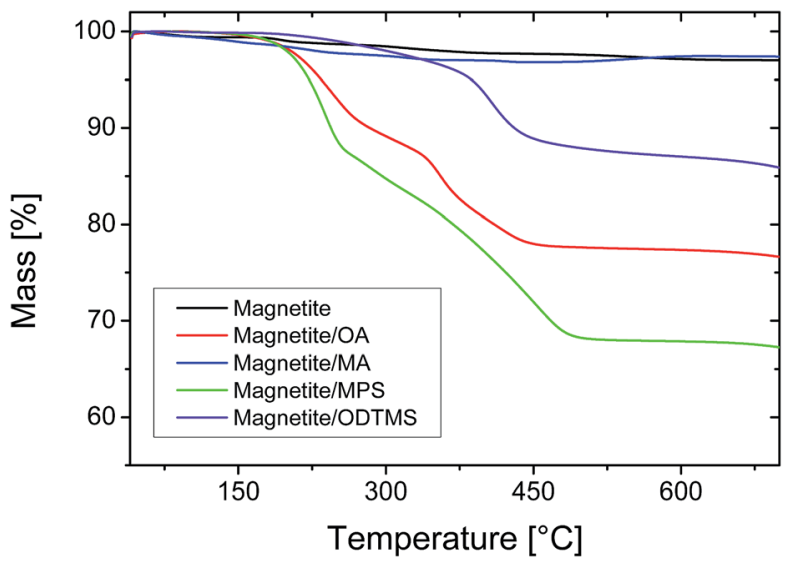

Fig. 1 Thermogravimetric analysis of magnetite nanoparticles with different surface functionalizations. is one of the most common functionalization strategies. ${ }^{30}$ The carboxylic acid group has a high affinity to the iron atoms and acts as a chelating agent. As stated in the Introduction, although encapsulation of oleic-acid-functionalized magnetite particles in miniemulsion is possible, the structure of these materials is in most cases a Janus-like morphology. A variation of the surface functionality can be helpful to achieve a homogeneous distribution of the particles in the polymer. ${ }^{16}$

\section{Surface functionalization of magnetite nanoparticles}

Superparamagnetic magnetite nanoparticles with a size of around $5 \mathrm{~nm}$ were synthesized by co-precipitation in the presence of oleic acid. The surface functionalization with oleic acid was successful, as proven by the thermogravimetric analysis (TGA) traces shown in Fig. 1. As already demonstrated in previous work from our group using silica nanoparticles, the structure of hybrid particles depends on the surface functionalization. ${ }^{16}$ A polymerizable methacrylic acid ester leads to a homogeneous distribution of the inorganic particles in the polymer, while a long alkyl chain gives a Janus-like structure. To achieve similar results with magnetite particles, oleic acid was replaced by methacrylic acid (MA) as a functionalization agent. However, this functionalization was not successful, as observed from the data shown in Fig. 1. We assume that the solubility of methacrylic acid in water is too high and the adsorption to the magnetite surface is not favorable. To prove that the reactive group of methacrylic acid and the double bond of oleic acid have no influence on the functionalization reaction, we carried out analogous experiments with stearic acid and propionic acid. The thermal decomposition of magnetite particles functionalized with stearic acid and propionic acid (shown in ESI, Fig. S1 $\dagger$ ) is similar to the particles functionalized with oleic and methacrylic acid: functionalization is only successful (high amount of organic material), with very hydrophobic acids but not with more hydrophilic ones.

Table 1 Characteristics of the samples presented in this work

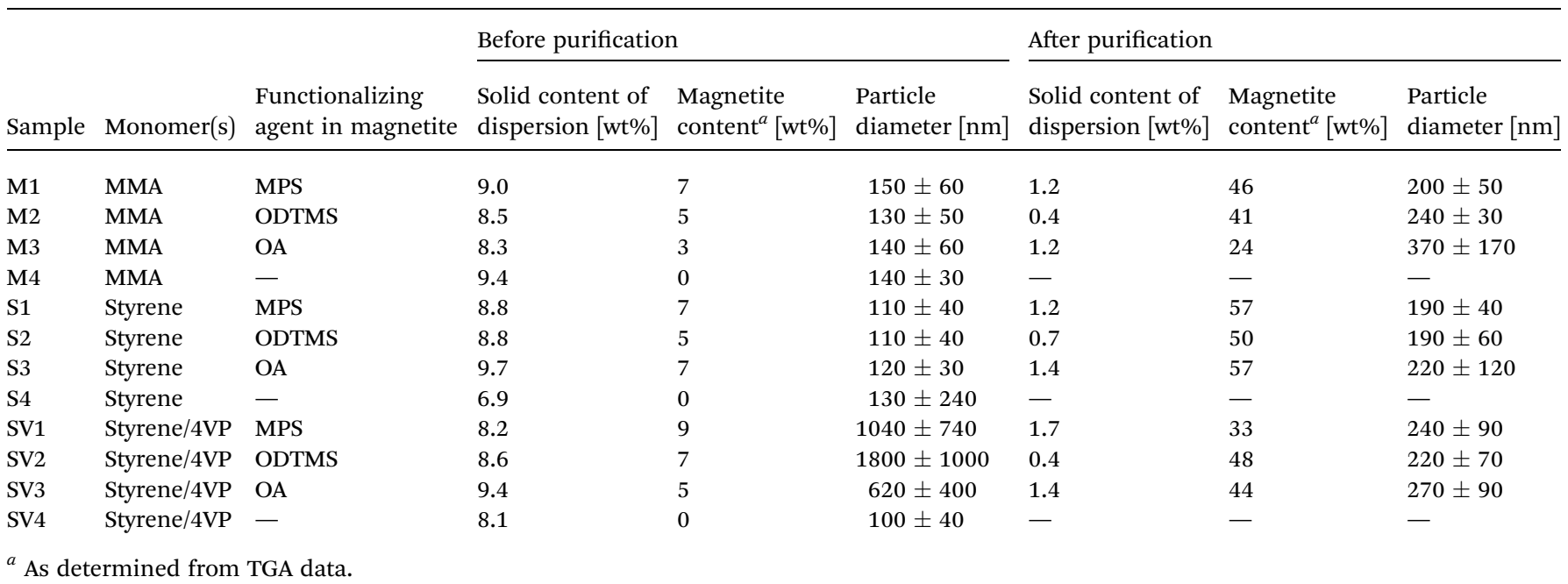


A possible alternative to carboxylic acids is the functionalization with trimethoxysilane compounds, which offer a relatively straightforward and facile way for surface functionalization. Many different reactive groups and chain lengths are accessible and facilitate the tuning of the properties of the functionalized particles. ${ }^{37}$ For the functionalization of the superparamagnetic magnetite particles, we used an analogous method to that reported by Bourgéat-Lami et $a l^{21}$ for silica nanoparticles. Condensation of the precursors leads to the formation of a covalent attachment of the functionalization agent around the particles, ${ }^{\mathbf{4}}$ so that removal during purification or polymerization is effectively avoided. As functionalization agents, 3-methacryloxypropyl trimethoxysilane (MPS) and octadecyl trimethoxysilane (ODTMS) were used, in a similar fashion to previous work from our group with silica particles. ${ }^{15}$ The MPS content of the functionalized particles is $30 \mathrm{wt} \%$, while the amount of organic materials on the ODTMS-functionalized particles is only $10 \mathrm{wt} \%$ (Fig. 1). This difference can be explained by a higher solubility of MPS in the reaction mixture. Before adsorption on the particles, the trimethoxysilanes hydrolyze and form oligomers. ${ }^{43}$ As ODTMS is much more hydrophobic than MPS, the oligomers should precipitate at much shorter chain lengths and, therefore, not be available anymore for surface functionalization. This behavior can be macroscopically observed during the reaction by an increase of turbidity in the reaction mixture. The precipitated functionalization agents were removed after functionalization by magnetic purification.

\section{Composition of hybrid materials}

The differently functionalized magnetite particles were encapsulated by miniemulsion polymerization. Since the polarity of the polymer plays a major role for the structure of the resulting hybrid material, ${ }^{\mathbf{1 2} 28}$ we chose methyl methacrylate (MMA) and styrene as monomers with slightly different polarities. Additionally, we used 4-vinylpyridine (4VP) as a co-monomer because it provides acid-base interactions with the surface of the inorganic particle and helps to increase the affinity between polymer and inorganic material. ${ }^{44}$ Miniemulsions containing a mixture of MMA and $4 \mathrm{VP}$ were not stable. For all further experiments, only the samples with pure MMA (labeled as M), pure styrene (labeled as S) or a mixture of styrene and $4 \mathrm{VP}$ (labeled as SV) are discussed.

After polymerization, the samples were filtered to remove coagulates. The samples were purified by using a magnet to collect the hybrid particles containing magnetite. The supernatant (i.e., non-magnetic dispersions) was removed and replaced with water. To evaluate the efficiency of the purification process, the solid content of the dispersions (determined by lyophilization) and the magnetite content of the lyophilized particles (measured by TGA) were determined before and after the purification step. Particle sizes were also analyzed before and after purification by dynamic light scattering (DLS). The results of these measurements are shown in Table 1 and Fig. 2. Since TGA measurements were conducted under a nitrogen atmosphere, the organic material did not oxidize completely,
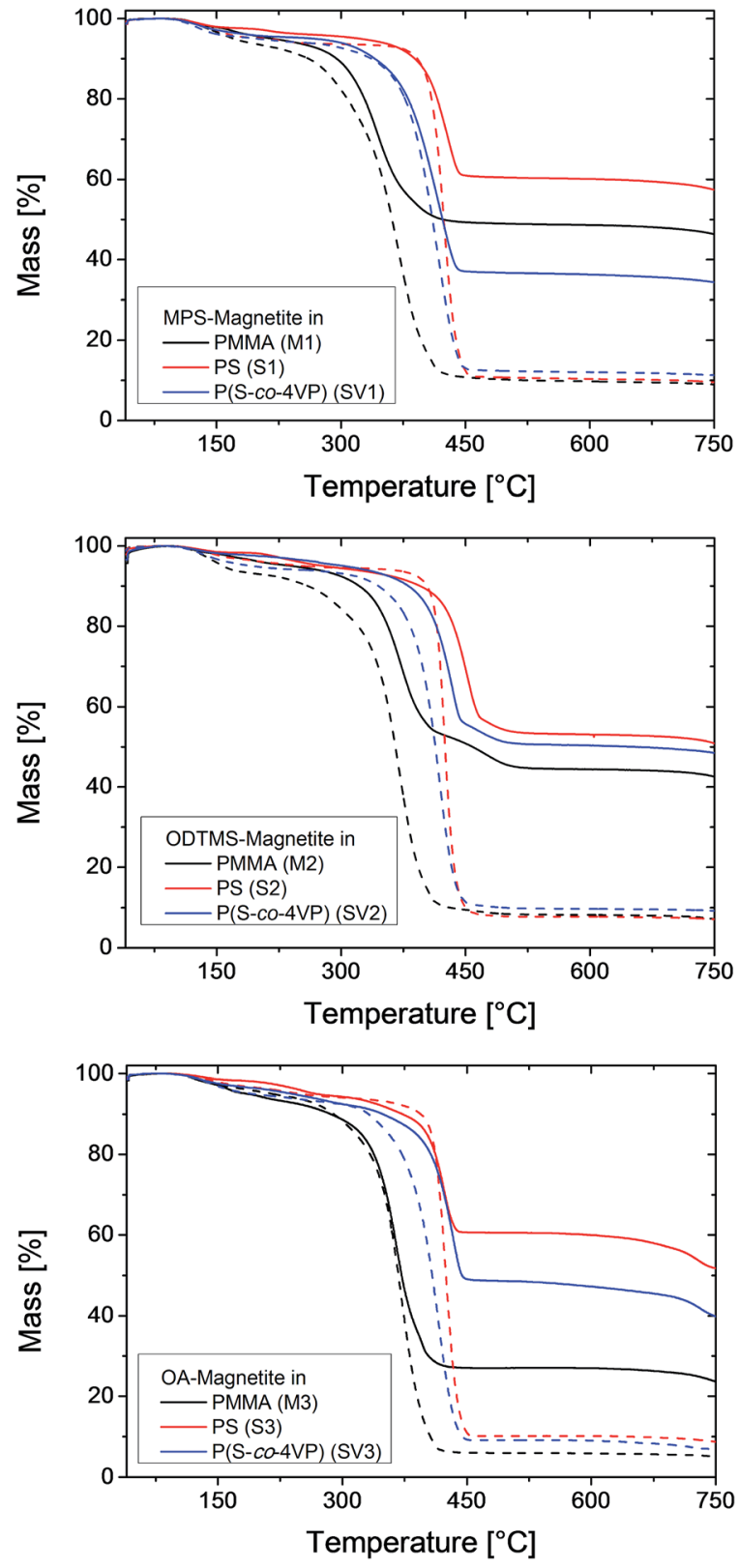

Fig. 2 TGA traces of hybrid nanoparticles containing differently functionalized magnetite nanoparticles and different polymers. The dashed lines are the samples before magnetic purification.

leaving a carbon residue. Accordingly, pure polymer samples (M4, S4, SV4) show a residual mass of $3 \mathrm{wt} \%$.

Directly after polymerization and filtration, the solid content of the samples was around $9 \mathrm{wt} \%$, which is close to the theoretical value of $10 \mathrm{wt} \%$. The miniemulsions were stable and showed only a small amount of coagulum after polymerization. After purification, the solid content is approximately $1.5 \mathrm{wt} \%$ for the samples containing MPS-functionalized and OAfunctionalized magnetite, and around $0.5 \mathrm{wt} \%$ for the samples with ODTMS-functionalized magnetite. These values indicate that only about $12 \%$ of the particles were sufficiently magnetic to be collected by the magnet. The rest were either pure polymer particles or contained only a low amount of 
magnetite. This observation is also in accordance with the electron micrographs of the samples before purification, as shown in Fig. 3-5. The inhomogeneous distribution of magnetite in the sample can be explained from the emulsification process. The preparation of the miniemulsion was achieved by ultrasonication of a macroemulsion. A high content of solid particles in a droplet leads to an increased viscosity, which hinders deformation and droplet breakup. ${ }^{45}$ As a consequence, empty particles are much more likely to be broken up than particles containing magnetite. After emulsification, the samples consist of a large number of small, empty particles besides some larger particles filled with magnetite. DLS data (see Table 1) before and after purification support this interpretation. Analogous effects had been previously observed for the encapsulation of silica particles. ${ }^{15,16,45}$ A major advantage of magnetite is that the inhomogeneous distribution in the system can be easily overcome by magnetic purification.

After synthesis, the samples should contain a theoretical amount of magnetite of $10 \mathrm{wt} \%$. With the residual polymer in TGA measurements taken into account, the expected result for the magnetite content (MC) in the TGA measurements should be about $13 \mathrm{wt} \%$. In most of the samples, between $40 \%$ and $55 \%$

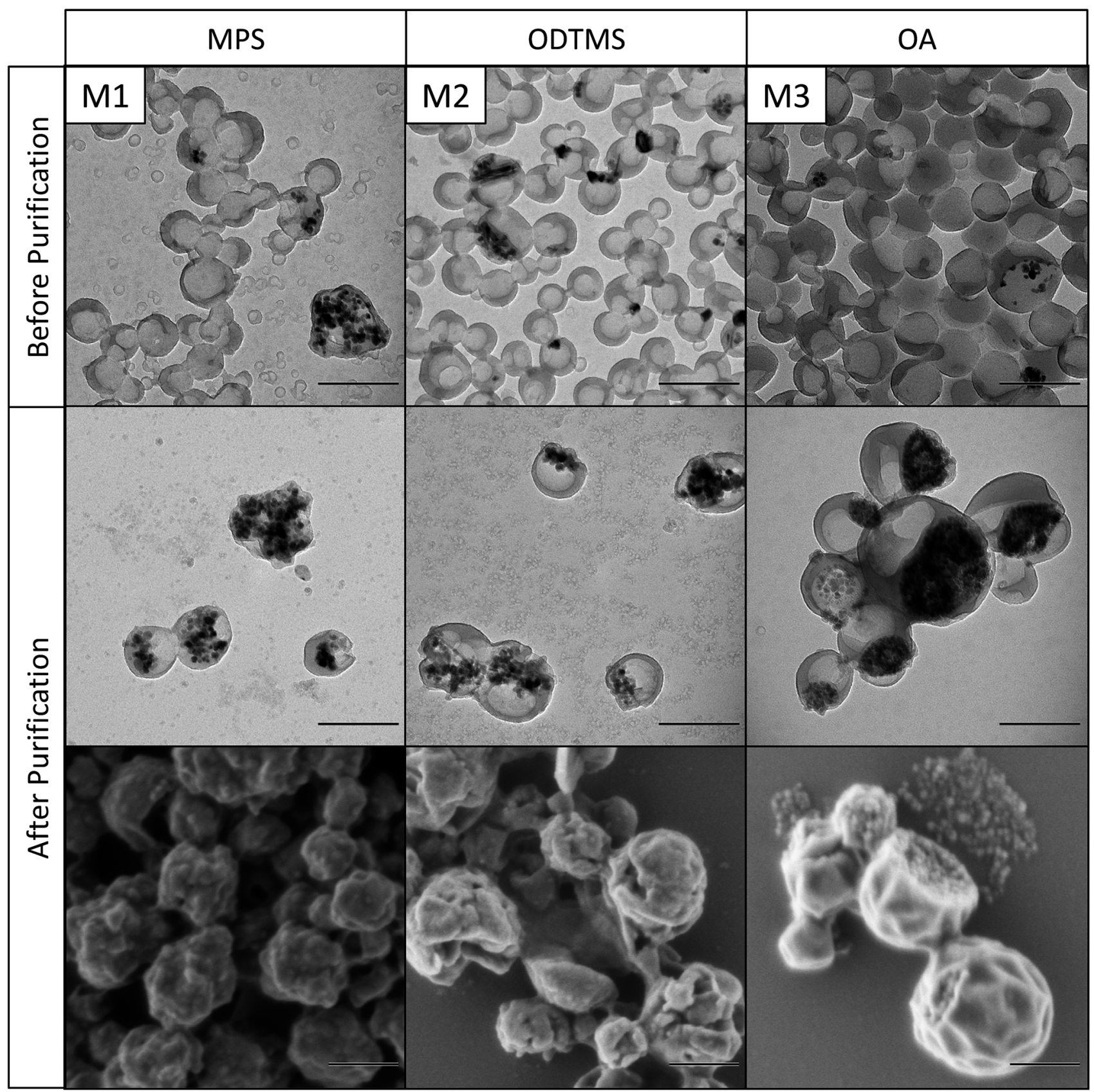

Fig. 3 Transmission and scanning electron micrographs of differently functionalized magnetite nanoparticles in PMMA before and after magnetic purification. Scale bars are $200 \mathrm{~nm}$. 


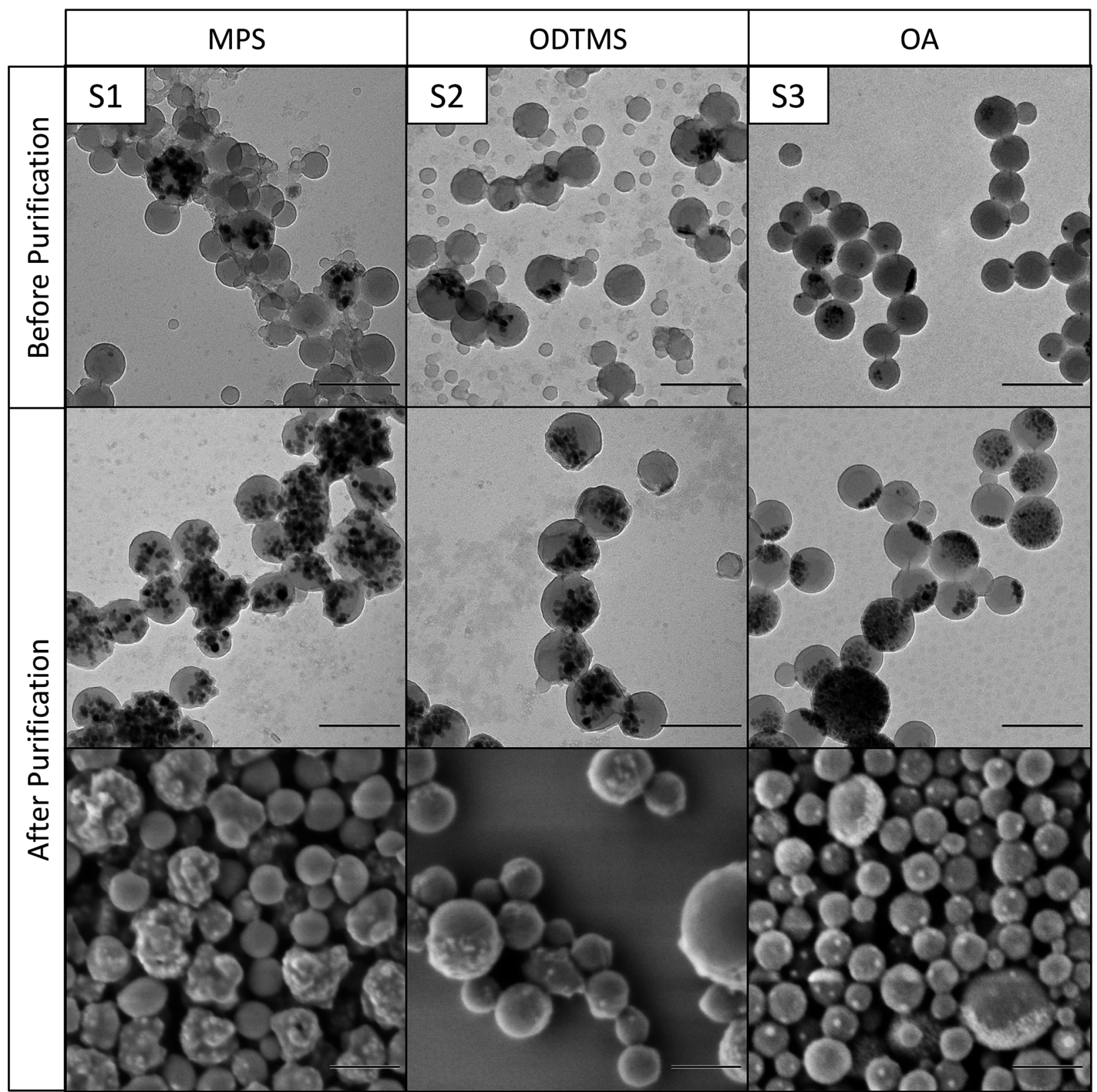

Fig. 4 Transmission and scanning electron micrographs of differently functionalized magnetite nanoparticles in polystyrene before and after magnetic purification. Scale bars are $200 \mathrm{~nm}$.

of the magnetite was encapsulated and is still present in the sample after filtration, while the rest was removed beforehand as a part of the coagulum. The higher encapsulation efficiency of $70 \%$ (sample SV1) was obtained for MPS-functionalized magnetite in poly(styrene-co-4VP), while the lowest amount (about 23\%) was found for OA-functionalized magnetite in MMA (sample M3).

As expected, the compositions of the dispersions change dramatically after magnetic purification. Due to removal of the pure polymer particles, the samples retain only the highly magnetic particles, which leads to a decrease in the solid content, as already stated above. In contrast, the magnetite content of the particles is much higher after purification (40 to $60 \mathrm{wt} \%$ ).

\section{Structure of hybrid nanoparticles}

The morphology of the hybrid samples as a function of the polymer and the surface chemistry of the magnetic nanoparticles was studied by electron microscopy. Fig. 3 shows magnetite nanoparticles encapsulated in PMMA, the nanoparticles in Fig. 4 are encapsulated in polystyrene, and Fig. 5 shows nanoparticles in poly(styrene-co-4VP). It needs to be noted that PMMA particles need to be additionally coated with carbon before TEM measurements, because they are very 


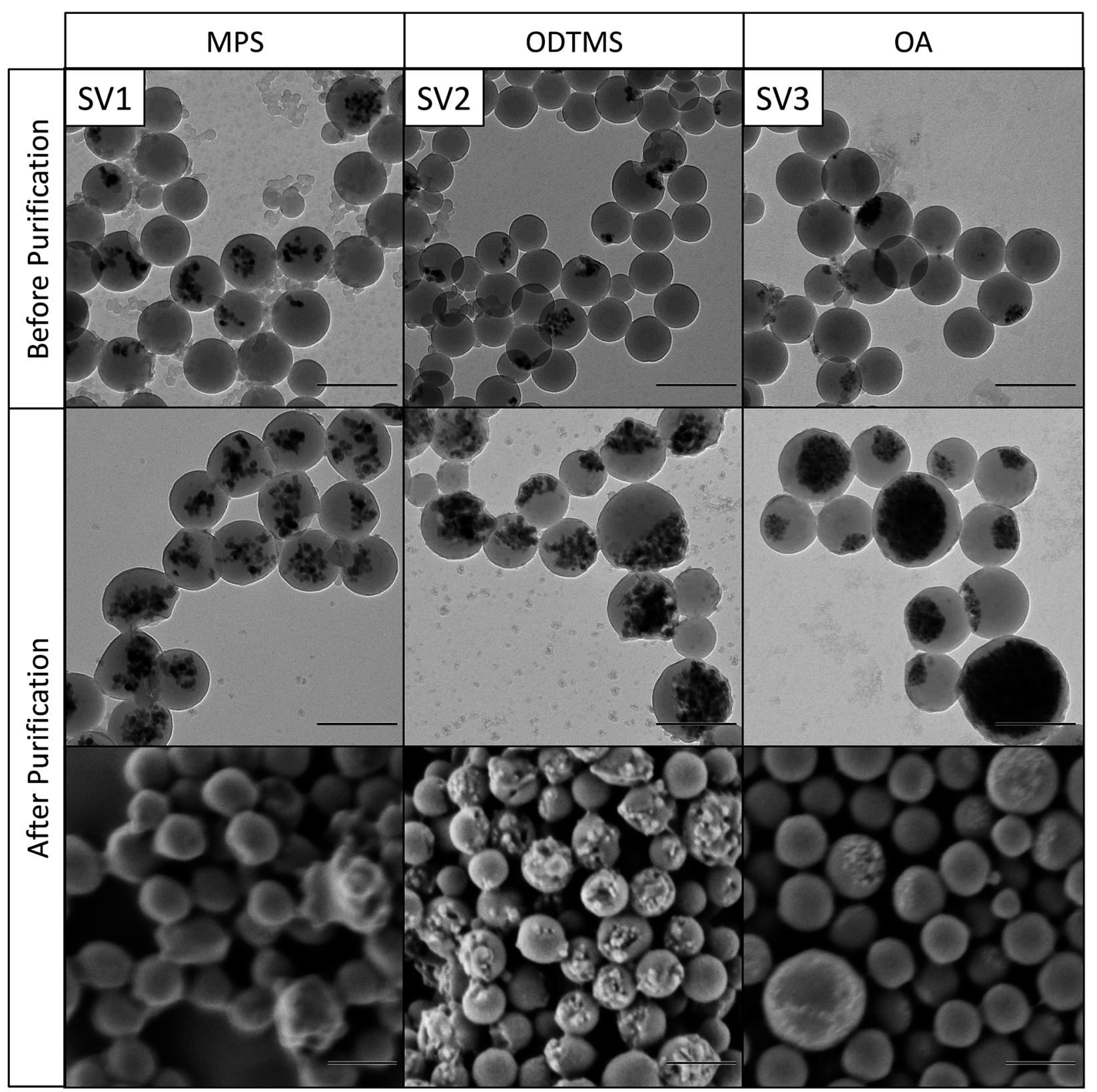

Fig. 5 Transmission and scanning electron micrographs of differently functionalized magnetite nanoparticles in poly(styrene-co-4VP) before and after magnetic purification. Scale bars are $200 \mathrm{~nm}$.

sensitive under the electron beam. This additional carbon layer appears in the TEM images as a shell, which is a result of the sample preparation. Polystyrene particles, much less sensitive, did not require this special treatment.

As already observed by TGA, the magnetite content of the hybrids increases very significantly after magnetic purification. The structure of the particles depends mainly on the applied surface functionalization. The first columns of Fig. 3-5 (M1, S1, SV1) show hybrids containing MPS-functionalized magnetite. In this case, the magnetite particles are distributed homogeneously inside the polymer. Especially in PMMA (M1), the particles are fully loaded and have lost their anisotropic shape due to the high amount of encapsulated magnetite. The structures of the particles containing ODTMS-functionalized magnetite (second columns, M2, S2, SV2) show a Janus-like structure. The magnetite particles are agglomerated only at one side of the hybrids, while the other side consists of pure polymer. Sample SV2 shows an "open shell" structure, where the magnetite seems to be trapped in a pocket formed by the polymer. This behavior indicates a very low affinity between polymer and ODTMS-functionalized magnetite. With OAfunctionalized magnetite, the structures are quite similar (M3, S3, SV3). All samples show a Janus morphology. In PMMA (M3), a high amount of free magnetite besides open polymer pockets can be found, which also indicates a low affinity between polymer and functionalized magnetite in this case. 
In general, the morphology of hybrid particles is controlled by a minimization of the interfacial energies in the system. ${ }^{12,27}$ Since all other parameters are kept constant in our experiments, the difference in structure is controlled by the different polarities of the different functionalization agents and polymers. ODTMS and OA possess a long alkyl chain and are much more hydrophobic than MPS, which results in a higher affinity to hydrophobic monomers like styrene, while MPS is more compatible with the polar MMA. In consequence, more hydrophobic particles functionalized with ODTMS and OA should be expected to be preferably inside the monomer to reduce the magnetite/water interface as much as possible, which is not the case. As the structures of the hybrids with differently functionalized magnetite are basically the same for all polymers, this hypothesis does not explain our observations. Theoretical calculations by Gonzalez-Ortiz and Asua describe structure control as a function of the different interfacial tensions in the system. ${ }^{12,27}$ However, this model is limited to systems in thermal equilibrium. In our system, MPS is able to copolymerize with the surrounding monomer, ${ }^{15}$ leading to a kinetic fixation of the MPS-functionalized particles inside the hybrids, thus avoiding formation of the thermodynamically preferred Janus structure.

\section{Conclusions}

We have demonstrated that the functionalization of magnetite nanoparticles with trialkoxy silanes is an efficient alternative to the classical method using oleic acid when the aim is to make them compatible with a polymer matrix. The versatility of the functionalization agents gives various possibilities to tune the surface properties of magnetite particles and, therefore, to control the morphology of hybrid particles in encapsulation experiments. While MPS-functionalized magnetite is homogeneously distributed in the polymer, ODTMS- and OAfunctionalized magnetite both form Janus structures. Magnetic purification of the dispersions increases the magnetite content up to $60 \mathrm{wt} \%$ in polymers with different polarities. Our work shows that silanization offers a tailorable surface functionalization that can help to tune the properties of hybrid particles according to the desired structure and material combination.

\section{Acknowledgements}

ADK acknowledges the financial support of his summer internship by the RISE program of the DAAD. RME acknowledges the financial support from the Spanish Ministry of Economy and Competitiveness through a Ramón y Cajal grant (grant No. RYC-2013-13451).

\section{Notes and references}

1 H. Zou, S. Wu and J. Shen, Chem. Rev., 2008, 108, 3893-3957. 2 H. Wang, P. Xu, S. Meng, W. Zhong, W. Du and Q. Du, Polym. Degrad. Stab., 2006, 91, 1455-1461.

3 G. H. Gao, G. H. Im, M. S. Kim, J. W. Lee, J. Yang, H. Jeon, J. H. Lee and D. S. Lee, Small, 2010, 6, 1201-1204.
4 Y. Mori and H. Kawaguchi, Colloids Surf., B, 2007, 56, 246254.

5 A. Jordan, P. Wust, R. Scholz, B. Tesche, H. Fähling, T. Mitrovics, T. Vogl, J. Cervós-Navarro and R. Felix, Int. J. Hyperthermia, 1996, 12, 705-722.

6 D. Wang, J. Zhu, Q. Yao and C. A. Wilkie, Chem. Mater., 2002, 14, 3837-3843.

7 E. Duguet, M. Abboud, F. Morvan, P. Maheu and M. Fontanille, Macromol. Symp., 2000, 151, 365-370.

8 X. Huang and W. J. Brittain, Macromolecules, 2001, 34, 32553260.

9 E. Bourgeat-Lami and J. Lang, J. Colloid Interface Sci., 1998, 197, 293-308.

10 F. Corcos, E. Bourgeat-Lami, C. Novat and J. Lang, Colloid Polym. Sci., 1999, 277, 1142-1151.

11 A. R. Mahdavian, M. Ashjari and A. B. Makoo, Eur. Polym. J., 2007, 43, 336-344.

12 J. M. Asua, Macromol. Chem. Phys., 2014, 215, 458-464.

13 P. J. Colver, C. A. L. Colard and S. A. F. Bon, J. Am. Chem. Soc., 2008, 130, 16850-16851.

14 S. Reculusa, C. Poncet-Legrand, S. Ravaine, C. Mingotaud, E. Duguet and E. Bourgeat-Lami, Chem. Mater., 2002, 14, 2354-2359.

15 A. Schoth, E. S. Adurahim, M. A. Bahattab, K. Landfester and R. Muñoz-Espí, Macromol. React. Eng., 2016, 10, 47-54.

16 A. Schoth, C. Wagner, L. L. Hecht, S. Winzen, R. Muñoz-Espí, H. P. Schuchmann and K. Landfester, Colloid Polym. Sci., 2014, 292, 2427-2437.

17 M. A. Hood, M. Mari and R. Muñoz-Espí, Materials, 2014, 7, 4057-4087.

18 K. Landfester, N. Bechthold, F. Tiarks and M. Antonietti, Macromolecules, 1999, 32, 5222-5228.

19 N. Jagielski, S. Sharma, V. Hombach, V. Mailänder, V. Rasche and K. Landfester, Macromol. Chem. Phys., 2007, 208, 2229-2241.

20 F. Tiarks, K. Landfester and M. Antonietti, Langmuir, 2001, 17, 5775-5780.

21 E. Bourgeat-Lami, N. N. Herrera, J. L. Putaux, S. Reculusa, A. Perro, S. Ravaine, C. Mingotaud and E. Duguet, Macromol. Symp., 2005, 229, 32-46.

22 L. P. Ramírez and K. Landfester, Macromol. Chem. Phys., 2003, 204, 22-31.

23 Y. Gao, Y. Song and Q. Zheng, Colloids Surf., A, 2012, 411, 4049.

24 K. Y. van Berkel and C. J. Hawker, J. Polym. Sci., Part A: Polym. Chem., 2010, 48, 1594-1606.

25 X. Qiao, M. Chen, J. Zhou and L. Wu, J. Polym. Sci., Part A: Polym. Chem., 2007, 45, 1028-1037.

26 M. Urban, A. Musyanovych and K. Landfester, Macromol. Chem. Phys., 2009, 210, 961-970.

27 L. J. Gonzalez-Ortiz and J. M. Asua, Macromolecules, 1995, 28, 3135-3145.

28 T. Staudt, T. O. Machado, N. Vogel, C. K. Weiss, P. H. H. Araujo, C. Sayer and K. Landfester, Macromol. Chem. Phys., 2013, 214, 2213-2222.

29 T. Gong, D. Yang, J. Hu, W. Yang, C. Wang and J. Q. Lu, Colloids Surf., A, 2009, 339, 232-239. 
30 K. G. Neoh and E. T. Kang, Polym. Chem., 2011, 2, 747-759. 31 S. Lu and J. Forcada, J. Polym. Sci., Part A: Polym. Chem., 2006, 44, 4187-4203.

32 S. Lu, J. Ramos and J. Forcada, Langmuir, 2007, 23, 1289312900.

33 F. Yan, J. Li, J. Zhang, F. Liu and W. Yang, J. Nanopart. Res., 2008, 11, 289-296.

34 H. Xu, L. Cui, N. Tong and H. Gu, J. Am. Chem. Soc., 2006, 128, 15582-15583.

35 W. Zheng, F. Gao and H. Gu, J. Magn. Magn. Mater., 2005, 288, 403-410.

36 M. B. Bannwarth, S. W. Kazer, S. Ulrich, G. Glasser, D. Crespy and K. Landfester, Angew. Chem., Int. Ed., 2013, 52, 1010710111.

37 R. P. Bagwe, L. R. Hilliard and W. Tan, Langmuir, 2006, 22, 4357-4362.

38 R. A. Bini, R. F. C. Marques, F. J. Santos, J. A. Chaker and M. Jafelicci Jr, J. Magn. Magn. Mater., 2012, 324, 534-539.
39 C. Atila Dinçer, N. Yıldız, N. Aydoğan and A. Çalımlı, Appl. Surf. Sci., 2014, 318, 297-304.

40 D. Li, W. Y. Teoh, D. Djunaedi, J. J. Gooding, C. Selomulya and R. Amal, Adv. Energy Mater., 2010, 12, B210-B214.

41 M. Bloemen, W. Brullot, T. T. Luong, N. Geukens, A. Gils and T. Verbiest, J. Nanopart. Res., 2012, 14, 1-10.

42 M. Kačenka, O. Kaman, S. Kikerlová, B. Pavlů, Z. Jirák, D. Jirák, V. Herynek, J. Černý, F. Chaput, S. Laurent and I. Lukeš, J. Colloid Interface Sci., 2015, 447, 97-106.

43 A. P. Philipse and A. Vrij, J. Colloid Interface Sci., 1989, 128, 121-136.

44 M. J. Percy, C. Barthet, J. C. Lobb, M. A. Khan, S. F. Lascelles, M. Vamvakaki and S. P. Armes, Langmuir, 2000, 16, 69136920.

45 L. L. Hecht, T. Merkel, A. Schoth, C. Wagner, K. Köhler, R. Muñoz-Espí, K. Landfester and H. P. Schuchmann, Chem. Eng. J., 2013, 229, 206-216. 